\title{
BtPDR: Bluetooth and PDR-Based Indoor Fusion Localization Using Smartphones
}

\author{
Yingbiao Yao, Qiaojing Bao, Qi Han, Ruili Yao, Xiaorong Xu, Junrong Yan \\ School of communication Engineering, Hangzhou Dianzi University \\ Hangzhou, Zhejiang 310018 - China \\ [e-mail: yaoyb@hdu.edu.cn] \\ *Corresponding author: Junrong Yan
}

Received December 22, 2016; revised August 20, 2017; accepted April 6, 2018;

published August 31, 2018

\begin{abstract}
:
This paper presents a Bluetooth and pedestrian dead reckoning (PDR)-based indoor fusion localization approach (BtPDR) using smartphones. A Bluetooth and PDR-based indoor fusion localization approach can localize the initial position of a smartphone with the received signal strength (RSS) of Bluetooth. While a smartphone is moving, BtPDR can track its position by fusing the localization results of PDR and Bluetooth RSS. In addition, BtPDR can adaptively modify the parameters of PDR. The contributions of BtPDR include: a Bluetooth RSS-based Probabilistic Voting (BRPV) localization mechanism, a probabilistic voting-based Bluetooth RSS and PDR fusion method, and a heuristic search approach for reducing the complexity of BRPV. The experiment results in a real scene show that the average positioning error is $<2 \mathrm{~m}$, which is considered adequate for indoor location-based service applications. Moreover, compared to the traditional PDR method, BtPDR improves the location accuracy by $42.6 \%$, on average. Compared to state-of-the-art Wireless Local Area Network (WLAN) fingerprint + PDR-based fusion indoor localization approaches, BtPDR has better positioning accuracy and does not need the same offline workload as a fingerprint algorithm.
\end{abstract}

Keywords: indoor localization; pedestrian dead reckoning; Bluetooth; received signal strength; probabilistic voting

This work was supported by National Natural Science Foundation of China (Grant No. 61100044, 61671192), the Program of Zhejiang Province Natural Science Foundation (Grant No. LY15F010008, LY16F010012), and the 2017 Open Research Foundation of Electronics Science and Technology the Top-ranking Discipline A Class in Zhejiang Province. 


\section{Introduction}

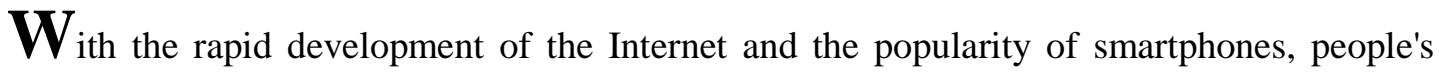
demand for location-based services (LBS) is gradually increasing [1]. Location-based services are widely used in many fields including people's work, business and military [2-3]. The key to LBS is its ability to accurately obtain the real-time position of users. Although smartphones can easily obtain an outdoor position through the Global Positioning System (GPS), they cannot easily obtain an indoor position in a building due to the occlusion of walls. Because of this, high accuracy indoor localization has become a research topic of interest in recent years.

Common methods of smartphone indoor localization are based on Received Signal Strength (RSS), for example, centroid localization [4-5], trilateration or multilateral localization [6-9] and fingerprint localization [10-13]. Centroid localization outputs the centroid of beacon nodes as a positioning result. This method is simplistic with poor positioning accuracy [4-5]. Trilateration or multilateral localization requires knowing the distances from an unknown node to at least three beacon nodes and then utilizes the distance and the position of the beacon nodes to build localization equations. Finally, it uses the maximum likelihood method [6-7], the interior point method [8], linear programming [9] and other mathematical optimization methods to calculate the position of unknown nodes. The traditional fingerprint localization approach [10-13] involves offline and online phases. In the offline phase, the approach collects RSS fingerprints of different indoor locations to create a fingerprint database. In the online phase, RSS fingerprints are compared to the fingerprints in the database to estimate the position of unknown nodes. However, traditional fingerprint localization is limited due to the necessity of a lot of offline work and the challenge of fingerprint ambiguity. Zuwei et al. [20] proposed a peer-to-peer navigation system (ppNav), avoiding the requirements of pre-deployed location services and detailed floorplans. The drawback of offline training of the fingerprint-based approach has been addressed by recent crowdsourcing-based methods in [21, 22]. To reduce RSS fingerprint ambiguity, Kaishun et al. [23] proposed a novel approach called FILA, which leverages channel state information (CSI) to build a propagation model and fingerprinting system at the receiver.

The other method of smartphone indoor localization is based on pedestrian dead reckoning (PDR). The PDR-based method employs inertial measurement unit (IMU) sensors, such as the accelerometer, gyroscope or magnetometer embedded in a smartphone, to solve indoor localization $[14,19]$. Since the PDR localization method can only provide relative position information, and the localization error caused by IMU sensors may accumulate with increased walking distance [30], this method needs another assistant localization method. Therefore, the fusion localization approach, which is based on PDR and another localization method, has attracted the attention of academics [15-18]. Lyu-Han et al. [16] employed the 
maximum likelihood method to fuse fingerprint and PDR localization. Bonhyun et al. [17] employed Kalman filtering to also fuse fingerprint and PDR localization. Perttula et al. [18] used particle filter to fuse map-matching and PDR localization. However, collecting RSS fingerprints or indoor map information in [16-18] took a lot of time.

In this paper, a Bluetooth RSS and PDR-based fusion localization approach (BtPDR) for smartphone indoor localization was proposed. Although the widespread deployment of WLAN has made WLAN-based fingerprint positioning (WFP) popular, this study argues that the localization approaches, which are based on Bluetooth Low Energy (BLE), are useful in specific scenarios such as factories, indoor garages and shopping malls. Bluetooth Low Energy has the advantages of low cost, low power consumption and low penetration [24, $25]$. Due to the low penetration feature of BLE, BLE beacons in different rooms or on different floors do not affect each other. Thus, BLE can achieve accurate, three-dimensional indoor localization. The Bluetooth RSS and PDR-based fusion localization approach uses Bluetooth RSS to localize the initial position of a smartphone, then employs the fusion results of Bluetooth RSS and PDR to track the trace of it. Moreover, BtPDR can adaptively modify the parameters of PDR. To summarize, the contributions of BtPDR include:

$\diamond \quad$ a Bluetooth RSS-based Probabilistic Voting (BRPV) localization method to reduce the negative impact of RSS instability on localization accuracy;

$\diamond \quad \mathrm{a}$ BRPV and PDR fusion localization approach (BtPDR) to improve indoor localization accuracy;

$\diamond \quad$ a heuristic search method to reduce the computational complexity of BRPV.

Experiment results in the real scene show that BtPDR can provide meter-level positioning accuracy for users with common smartphone devices, which is considered adequate for indoor LBS applications. Specifically, BtPDR's average positioning errors in three real experimental scenes were $1.86 \mathrm{~m}, 1.94 \mathrm{~m}$ and $1.58 \mathrm{~m}$. Compared to the traditional PDR, BtPDR improves the positioning accuracy by $42.6 \%$, on average. In addition, compared to the state-of-the-art WLAN fingerprint + PDR-based fusion indoor localization algorithm, BtPDR has better positioning accuracy and does not need the same offline work.

\section{The traditional PDR localization}

The basic principle of PDR is shown in Fig. 1. First, PDR starts from a known location. Then, the heading and step length information of the walking pedestrian can be obtained by built-in sensors in a smartphone, for example, a magnetometer or an accelerometer. Using this information, the next position of the pedestrian is calculated as:

$$
\left\{\begin{array}{l}
x_{k+1}=x_{k}+L_{k} \times \sin \left(\theta_{k}\right) \\
y_{k+1}=y_{k}+L_{k} \times \cos \left(\theta_{k}\right)
\end{array}\right.
$$

Where $\left(x_{k}, y_{k}\right)$ is the current location, $\left(x_{k+1}, y_{k+1}\right)$ is the next location, $\theta_{k}$ is the heading, 
and $L_{k}$ is the step length.



Fig. 1. PDR algorithm schematic

When pedestrians are moving, an acceleration waveform, collected by the built-in acceleration sensor, is created, as shown in Fig. 2. Each cycle waveform in Fig. 2 represents a forward step. After filtering the high frequency components and limiting amplitude, the acceleration waveform is shown in Fig. 3. By using the zero-crossing detection method in [14], the number of walking steps was counted.

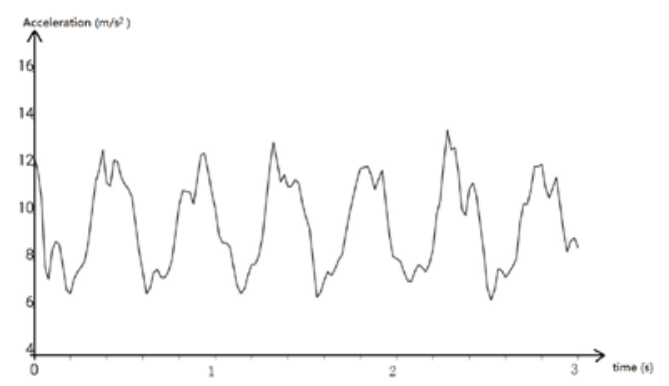

Fig. 2. Acceleration waveform during normal walking

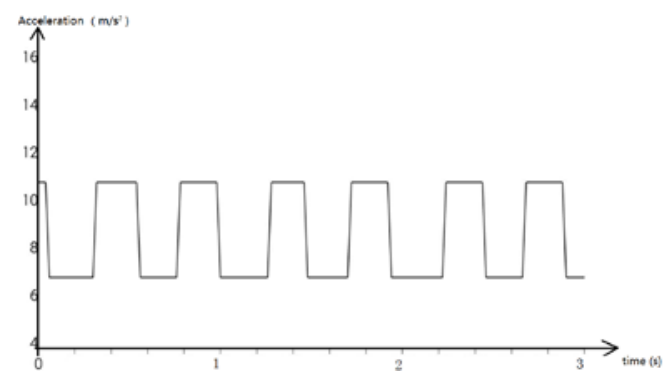

Fig. 3. Acceleration waveform after filtering

To estimate step size, under the condition of an acceleration sensor fixed in shoes, a step size formula was proposed in [14] as follows:

$$
L=K * \sqrt[4]{a_{\max }-a_{\min }}
$$


Where $K$ is a constant value, belonging to [0.2, 0.5], and is related to the gender, height and weight of the pedestrian and $a_{\max }$ and $a_{\min }$ denote the maximum and minimum acceleration values in a step obtained by the acceleration sensor. However, BtPDR uses built-in sensors and assumes that the pedestrian holds a smartphone in their hand. Therefore, for this experiment, the step size formula should be different than in [14]. According to the experiment results, BtPDR employs a step size formula as follows:

$$
L=K * \sqrt[4]{\left(a_{\max }-a_{\min }\right)+2}
$$

The main drawbacks of traditional PDR are as follows: 1) it is unable to determine the initial location; 2) it uses acceleration changes to estimate step size and the estimation error varies with the individual and 3) it is relative localization and cannot eliminate cumulative errors, hence, its cumulative error grows with increased walking distance.

\section{The proposed BRPV localization}

To overcome the shortcomings of traditional PDR, a BRPV to assist PDR to realize high-precision indoor localization was proposed.

\subsection{The RSS ranging model}

Due to the weak penetrability of Bluetooth radio, a smartphone and Bluetooth beacon are usually in the same enclosed space to communicate with each other. Therefore, the signal propagation path loss between the smartphone and Bluetooth beacon can be modeled with a log-normal distribution as shown in (4).

$$
P_{r, d b}(d)=P_{r, d b}\left(d_{0}\right)-10 \times n_{p} \times \log \left(d / d_{0}\right)+X
$$

Where $d_{0}$ is the reference distance; $d$ is the distance between the smartphone and Bluetooth beacon; $P_{r, d B}(d)$ is the path loss at $d_{0}$ distance to the Bluetooth beacon; $n_{p}$ is the path loss constant, usually equaling $2 \sim 6$ and $X$ is a Gaussian random variable that obeys $\mathrm{N}\left(0, \sigma^{2}\right)$. Then, according to (4), the distance $d$ can be obtained as follows:

$$
d=d_{0} \times 10^{\left(P_{r, d B}\left(d_{0}\right)-P_{r, d B}(d)\right) / 10 \times n_{p}}
$$

However, in the actual application, due to noise interference (e.g., multipath and shadow effect), $P_{r, d B}(d)$ has large fluctuations, meaning that the estimated distance may be far from the real distance. Therefore, traditional RSS range-based multilateral localization may produce large localization errors at times.

\subsection{The proposed BRPV}

To tolerate RSS instability, BRPV does not directly use RSS to compute the distance between the smartphone and Bluetooth beacon; rather, it uses RSS to compute the 
probability of a smartphone's true location in different areas. Specifically, BRPV uses the Bluetooth beacon as a central point to obtain multiple concentric rings then calculates the probability that the real position of the smartphone falls into each concentric ring. When there are multiple Bluetooth beacons, the concentric rings of different Bluetooth beacons will intersect to create multiple areas. Then the optimal area will be selected by using the probabilistic voting method on each Bluetooth beacon. Finally, the centroid of the optimal area will be regarded as the location result. Advantages of BRPV are that it has outstanding tolerance to RSS instability and it can output localization results with a degree of reliability.



Fig. 4. RSS mapping distance

The articles in [26-28] point out that an RSS at a fixed position generally obeys the Gaussian distribution of $\mathbf{N}\left(\mu, \sigma^{2}\right)$. To make a tradeoff between localization accuracy and RSS instability tolerance, BRPV defines four concentric rings, as shown in Fig. 4. The radiuses of these four rings are D1, D2, D3 and D4. These four rings divide the plane into five areas and the corresponding RSS ranges are $(-\infty, \mu-1.5 \sigma],(\mu-1.5 \sigma, \mu-0.5 \sigma],(\mu-0.5 \sigma, \mu+0.5 \sigma],(\mu+0.5 \sigma$, $\mu+1.5 \sigma]$ and $(\mu+1.5 \sigma,+\infty)$, where $\mu$ is the measured RSS and $\sigma$ is a pre-determined standard deviation. That is to say, D1, D2, D3 and D4 are obtained by (5), with $P_{r, d B}(d)$ equaling $\mu+1.5 \sigma, \mu+0.5 \sigma, \mu-0.5 \sigma$ and $\mu-1.5 \sigma$, respectively.

After the smartphone receives the broadcast of the Bluetooth beacon, the probability of its real position in these five areas can be calculated by (6), where dis $s_{\text {true }}$ is the true distance between the smartphone and the Bluetooth beacon. Then, the number of votes of each area can be decided according to corresponding probability of each area, as shown in Fig. 4. The probability of areas 5 and 1 is 0.067 and they are assigned one vote. The probability of areas 4 and 2 is 0.242 , about 3.6 times more than that of areas 5 and 1, hence, they are assigned four votes. The probability of area 3 is 0.383 , about 5.73 times more than that of areas 5 and 
1 , so area 3 is assigned six votes.

$$
P=\left\{\begin{array}{l}
P(\text { the smartphone is in area } 1)=P\left(\text { dis }_{\text {ture }}>D_{4}\right)=\phi(\mu-1.5 \sigma)=0.067 \\
P(\text { the smartphone is in area } 2)=P\left(D_{3}<\text { dis }_{\text {ture }}<D_{4}\right)=\phi(\mu-0.5 \sigma)-\phi(\mu-1.5 \sigma)=0.242 \\
P(\text { the smartphone is in area } 3)=P\left(D_{2}<\text { dis }_{\text {ture }}<D_{3}\right)=\phi(\mu+0.5 \sigma)-\phi(\mu-0.5 \sigma)=0.383 \\
P(\text { the smartphone is in area } 4)=P\left(D_{1}<\text { dis }_{\text {ture }}<D_{2}\right)=\phi(\mu+1.5 \sigma)-\phi(\mu+0.5 \sigma)=0.242 \\
P(\text { the smartphone is in area } 5)=P\left(\text { dis }_{\text {ture }}<D_{1}\right)=1-\phi(\mu+1.5 \sigma)=0.067
\end{array}\right.
$$

The specific localization process of BRPV is as follows:

Assume that the received RSS vector of the smartphone is $\overrightarrow{R S S}=\left(r s s_{k}^{1}, r s s_{k}^{2}, \ldots r s s_{k}^{N}\right)$, where $r s s_{k}^{i}$ denotes the RSS of the $i$-th Bluetooth beacon in the $k$-th step. According to $r s s_{k}^{i}$, the probability voting areas of the $i$-th Bluetooth beacon can be computed. An example of probability voting areas with three Bluetooth beacons is shown in Fig. 5. From Fig. 5 it can be seen that the probability voting areas of different Bluetooth beacons overlap each other, dividing the plane into more subareas. The number of votes for each subarea is equal to the sum of the number of votes polled by each Bluetooth beacon. The centroid of the subarea with the most votes is the final localization result. For example, if the shadowed subarea in Fig. 5 has the most votes, then the final localization result is its centroid.

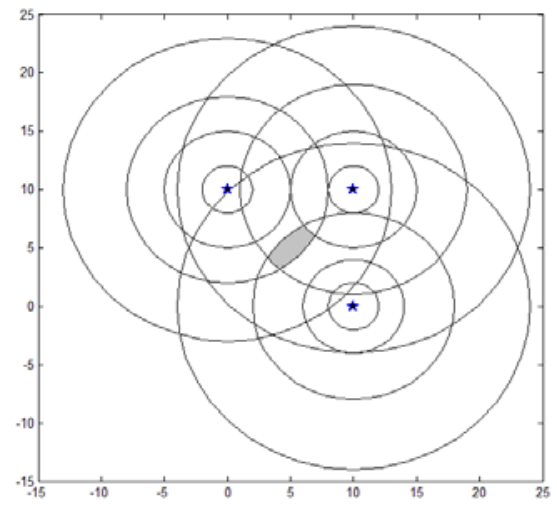

Fig. 5. Probability voting areas with three Bluetooth beacons

\subsection{A full search method for BRPV}

When there are many Bluetooth beacon nodes, the plane is divided into a lot of voting subareas, as shown in Fig. 5. In this case, it is very difficult to compute the boundary of each subarea and its centroid. Focusing on this problem, a simple, approximate calculation method, called a full search, was employed to derive the location with the most number of votes.

In the full search, a grid lattice to represent a subarea was employed, as shown in Fig. 6. For each grid point, votes were computed according to (7). The grid point with the most number of votes was used as the localization results of BRPV. If there were multiple grid points with the same number of votes, their averaged value was used as the localization results of BRPV. While the full search method has high computation complexity if the grid 
size is small, a smaller grid size can help to improve localization accuracy as the space is finely described. In this paper, a grid size of $0.1 \mathrm{~m}$ was chosen.

$$
\left(x_{r s s}, y_{\text {rss }}\right)=\arg \max _{(x, y)} V \equiv \sum_{i=1}^{m} v_{i}(x, y)
$$

Where $m$ is the number of Bluetooth beacons, $v_{i}(x, y)$ is the number of votes polled by the $i$-th Bluetooth beacon for the position $(x, y), V$ is the total number of votes polled by all Bluetooth beacons, and $\left(x_{r s s}, y_{r s s}\right)$ is the localization results of the full search method.

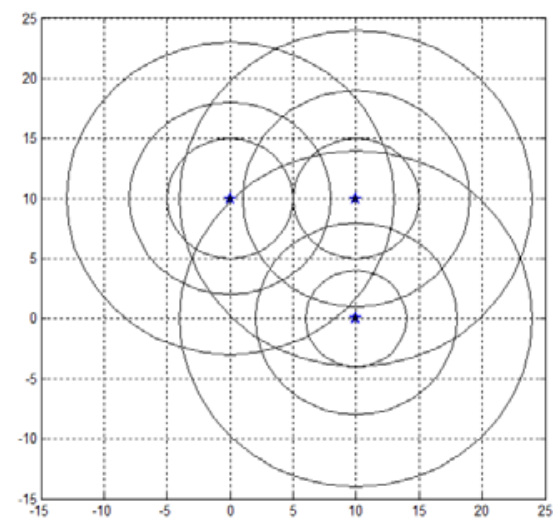

Fig. 6. The proposed full search scheme of BRPV

\subsection{A heuristic search method for BRPV}

Although the idea of the full search method is simple, and its localization accuracy is high, its complexity is also high, which limits its application with smartphones. Therefore, a heuristic search scheme for BRPV was proposed. The specific steps are as follows:

Step 1: Determine the initial search location $\boldsymbol{x} \boldsymbol{e}_{r s s}(0)=\boldsymbol{x} \boldsymbol{e}_{p d r}=\left(x_{p d r}, y_{p d r}\right)$. BRPV employs $\boldsymbol{x} \boldsymbol{e}_{p d r}$, the localization results of PDR, as the initial search position.

Step 2: Take $N$ equal interval points from the circle whose center is $\boldsymbol{x} \boldsymbol{e}_{r s s}(0)$ and radius is search_step, which is shown in Fig. 7. The coordinates of these $N$ search points are as follows:

$$
\begin{aligned}
& x e_{k}=x e_{0}+\text { seach_step } \times\left[\cos \theta_{k}, \sin \theta_{k}\right], \\
& \theta_{k}=(k-1) * \frac{2 \pi}{N}, \quad k=1,2, \cdots, N
\end{aligned}
$$

Step 3: Calculate the value of the objective function, defined in (9), at the above $N+1$ search points (including the circle center).

$$
f\left(x e_{k}\right)=\sum_{i=1}^{m} V_{i}\left(x e_{k}\right), \quad k=0,1, \cdots, N
$$

Step 4: Assume that there are $M$ positions which have the same maximum value $\boldsymbol{x}_{\text {rss_opt }}$ in the current search iteration. Then, BRPV updates $\boldsymbol{x} \boldsymbol{e}_{r s s}(0)$ and search_step according to (11) 
and (12) for the next search iteration.

$$
\begin{gathered}
x e_{\text {rss_opt }}=\max _{k=0,1, \cdots, N}\left\{f\left(x e_{k}\right)\right\} \\
x e_{\text {rss }}(0)=\frac{1}{M} \sum_{i=1}^{M} x e_{\text {rss_opt }}(i) \\
\text { seach_step }=\alpha^{*} \text { seach_step }
\end{gathered}
$$

Step 5: Repeat steps 2 4 until search_step is less than stop_th. The localization results of BRPV are the last updated $\boldsymbol{x} \boldsymbol{e}_{r s s}(0)$.

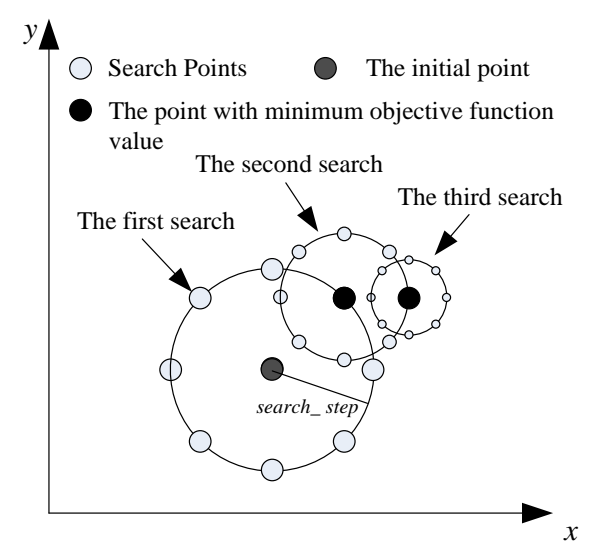

Fig. 7. The proposed heuristic search scheme of BRPV

In the previous BRPV, $\alpha$ is the convergence factor, which is used to control the convergence speed and, in theory, belongs to $(0,1)$ and stop_th is the search stopping threshold. The localization accuracy and complexity of BRPV are mainly determined by $\alpha$ and stop_th. Generally, the smaller stop_th is and the larger $\alpha$ is, the higher the localization accuracy and complexity will be.

In the simulation experiment, $N=16$, search_step $=5$, stop_th $=0.1$, and $\alpha=0.8$, meaning that BRPV needed to search approximately 306 points in each localization calculation. However, if the full search method with $0.1 \mathrm{~m}$ grid size described in Section 3.3 had been employed, BRPV would have needed to search approximately 15,000 points in each localization calculation with a location area of $6 \mathrm{~m} \times 25 \mathrm{~m}$. The number of search points of the proposed heuristic search method is only $2.04 \%$ that of the full search method.

\section{The proposed BtPDR localization}

\subsection{The specific flow of the BtPDR}

The traditional PDR can neither determine initial position nor eliminate cumulative error 
and estimate the step size accurately, and the localization accuracy of the traditional RSS range-based localization is low when each step receives few RSS samples. To solve the presented problems, a Bluetooth RSS and PDR-based fusion localization approach (BtPDR) for smartphone indoor localization and tracking systems was proposed. First, the initial position of a smartphone is localized with the proposed BRPV algorithm. Second, while the smartphone is moving, it is tracked with the traditional PDR and BRPV at the same time. Last, the proposed BtPDR can intelligently fuse PDR and BRPV localization results, adaptively modifying the parameters of PDR. The overall system architecture of BtPDR is shown in Fig. 8.

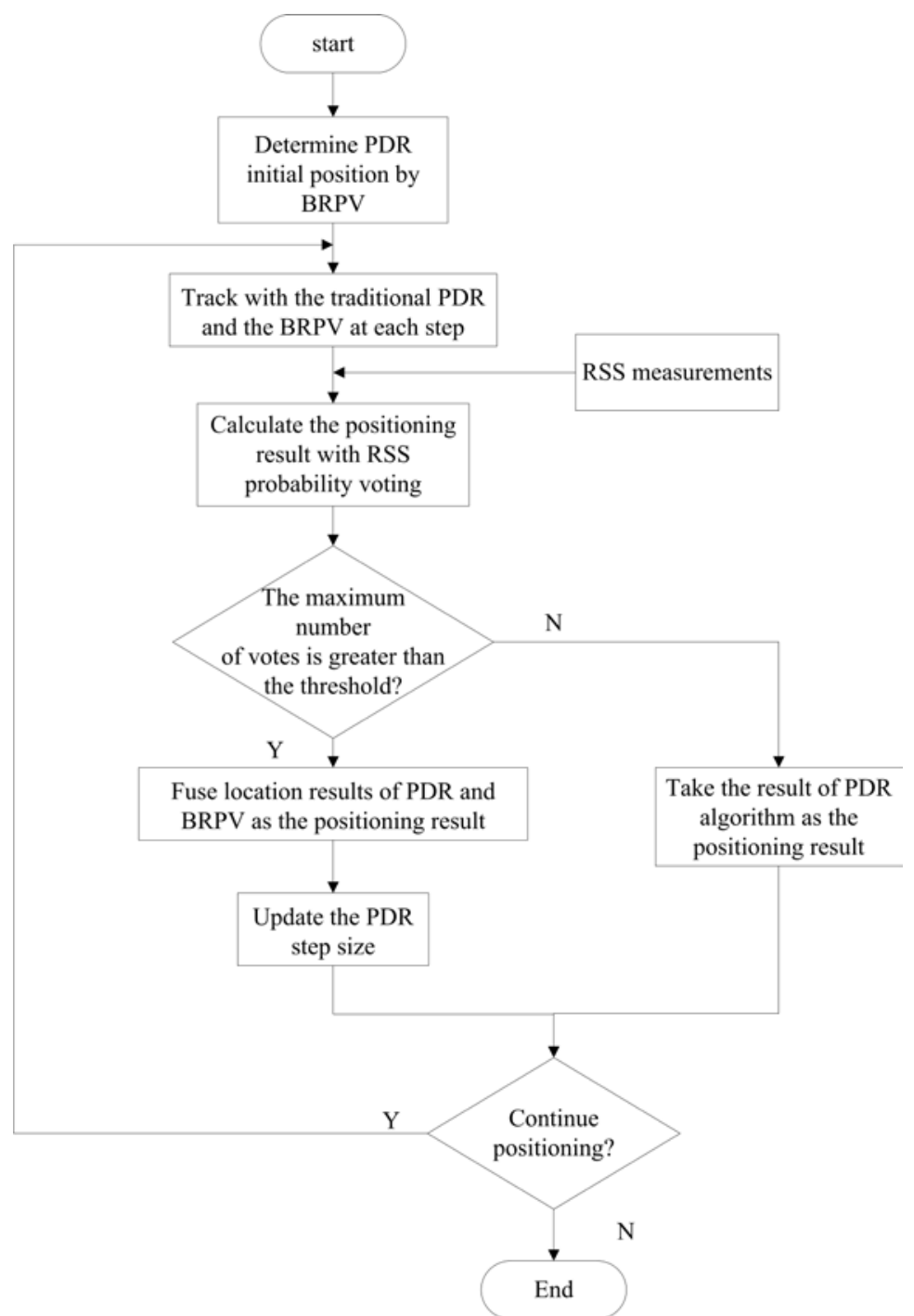

Fig. 8. Overall system architecture of BtPDR 


\subsection{Fusion rules of BtPDR}

During the smartphone tracking process, after $\boldsymbol{x} \boldsymbol{e}_{p d r}$, the positioning results of PDR, and $\boldsymbol{x} \boldsymbol{e}_{r s s}$, the positioning results of BRPV, are derived, the next key issue is how to fuse $\boldsymbol{x e}_{p d r}$ and $\boldsymbol{x} \boldsymbol{e}_{r s s}$. As mentioned, $\boldsymbol{x} \boldsymbol{e}_{r s s}$ is easily affected by the fluctuations of RSS and the number of votes at $\boldsymbol{x} \boldsymbol{e}_{r s s}$ reflects the probability that the actual position being in a region; the greater the number of votes, the greater the probability of the real location being in the region. Therefore, to reduce the influence of the RSS fluctuation, BtPDR discards $\boldsymbol{x} \boldsymbol{e}_{\text {rss }}$ with the number of votes less than or equal to a threshold $\beta$. Only when the number of votes $V_{\text {rss }}$ of BRPV is greater than $\beta$, then $\boldsymbol{x}_{\text {rss }}$ is believed to be reliable. When the results of PDR and BRPV need to be fused, the fusion is based on:

$$
x e=\left\{\begin{array}{cc}
\frac{V_{p d r}}{V_{r s s}+V_{p d r}} \cdot x e_{p d r}+\frac{V_{r s s}}{V_{r s s}+V_{p d r}} \cdot x e_{r s s} & V_{r s s}>\max \left\{\beta, V_{p d r}\right\} \\
x e_{p d r} & \text { otherwise }
\end{array}\right.
$$

Where $\boldsymbol{x} \boldsymbol{e}$ is the final fusion localization results and $V_{r s s}$ and $V_{p d r}$ are the number of votes by probabilistic voting at $x e_{r s s}$ and $x e_{p d r}$, respectively. It can be seen in (13) that the weighted value of $x e$ is the number of votes of $\boldsymbol{x} \boldsymbol{e}_{p d r}$ and $\boldsymbol{x} \boldsymbol{e}_{r s s}$. The idea behind the weighted fusion method is to avoid the emergence of large errors by limiting the correction range of positions, making the fusion results more reliable. Moreover, the initial position localized by BRPV only affects the accuracy at the beginning. After BtPDR fuses the localization results of PDR and BRPV several times, the localization accuracy will converge and be independent of the initial position.

\subsection{The parameter update of BtPDR}

After BtPDR fuses the localization results of PDR and BRPV, it needs to adaptively update the step size of PDR. In this study, it was assumed that $\left(x_{k}, y_{k}\right)$ are the coordinates of the $k$-th fusion results and $\left(x_{p d r}, y_{p d r}\right)$ are the coordinates of the current PDR. Then the step size correction ratio rate can be computed:

$$
\text { rate }=\frac{\sqrt{\left(x_{k-1}-x_{k}\right)^{2}+\left(y_{k-1}-y_{k}\right)^{2}}}{\sqrt{\left(x_{k-1}-x_{p d r}\right)^{2}+\left(y_{k-1}-y_{p d r}\right)^{2}}}
$$

When the rate is greater than 0.6 and less than 1.5, it is an effective correction. Next, BtPDR updates the $K$ in (3) as follows:

$$
\begin{gathered}
K_{\text {new }}=I_{r} * K \\
I_{r}=\left\{\begin{array}{cc}
\text { rate } & \text { rate } \in[0.6,1.5] \\
1 & \text { otherwise }
\end{array}\right.
\end{gathered}
$$




\section{Experimental results}

\subsection{The relationship between RSS and distance}

This experiment was used to derive the relationship between RSS and distance in an actual scene. The experimental environment was an indoor rectangular corridor with a few obstacles.

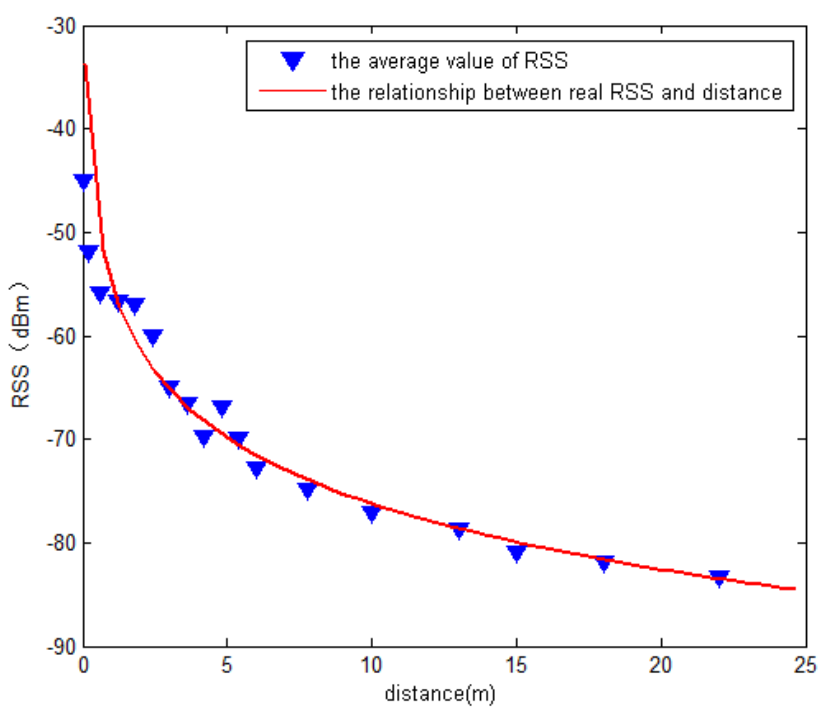

Fig. 9. The relationship between real RSS and distance

Bluetooth RSS data was collected by the smartphone at different distance from the Bluetooth beacon. At each location, 100 RSS acquisitions were carried out. Then, a Gaussian filter [29] was utilized to reduce the influence of small probability and big disturbance events on the overall measurement. The relationship between real RSS and distance by experiment is as shown in Fig. 9. The blue triangle in Fig. 9 is the average value of RSS after using a Gaussian filter and the red curve is the curve of (16).

Letting $d_{0}=1$, through the experiment the following values were determined: $P_{r, d B}\left(d_{0}\right)=$ $-55 \mathrm{dBm}, n_{p}=2.12$. The relationship between RSS and distance is then:

$$
R S S=-55-10 \times 2.12 \times \log (d)+X
$$

In the experiment, it was found that $X$ obeys a Gaussian distribution of $\mathbf{N}\left(0, \sigma^{2}\right)$ and $\sigma$ varies with the received position of the smartphone. In the following experiment, for simplicity, $\sigma=$ 7 was set and (16) was used to model all cases. It must be noted that 1) if only one measurement at one distance is made, the relationship between RSS and distance will not be as ideal as Fig. 9 and the error of the model in (16) will become bigger and 2) in the whole geographical space, the real relationship between RSS and distance does not comply with (16). Fortunately, experiment results show that the proposed BRPV and BtPDR can tolerate 
the suggested model errors, yielding satisfactory localization results.

\subsection{The performance analysis of BRPV}

To prove the localization accuracy of BRPV, simulations were carried out using MATLAB software. The simulation parameters are as follows: the number of total nodes is 104, including 4 Bluetooth beacons and 100 unknown nodes; the positions of the beacons are fixed and their coordinates are $(6,6),(0,12)(6,18)$, and $(0,24)$; the unknown nodes are uniformly distributed, in a fixed position, in a $6 \mathrm{~m} \times 25 \mathrm{~m}$ rectangular region and each unknown node acquires 10 RSS for each beacon node. The experimental results are the averaged results of 1000 experiments.

\subsubsection{The area division of BRPV}

This section analyzes the impact of the number of areas of BRPV. The plane is divided into three, five, seven, and nine areas, and the number of votes is assigned according to the probability that the smartphone falls into these areas. Simulation results as shown in Table 1, where BRPV uses the full search method in section 3.3. From Table 1, it can be determined that having three or nine areas is inappropriate. Compared to five areas, seven areas can improve localization accuracy by $1.76 \%$ at the cost of increasing CPU time by $26.4 \%$. Therefore, considering the localization accuracy and complexity, BRPV employed five areas in the following experiments.

Table 1. The impact of area division on algorithm performance

\begin{tabular}{|c|c|c|}
\hline The number of areas & CPU time per node (ms) & Absolute localization error (m) \\
\hline 3 & 50.1 & 6.732 \\
\hline 5 & 70.3 & 1.534 \\
\hline 7 & 88.9 & 1.507 \\
\hline 9 & 105.6 & 1.528 \\
\hline
\end{tabular}

\subsubsection{Compared BRPV to other algorithms}

In this section, BRPV is compared with the traditional least squares based multilateral positioning (LSMP) and fingerprint positioning (FP) methods. To do so, a fingerprint reference point was deployed every two meters, creating 52 reference points. For each reference point, 50 fingerprint acquisitions were carried out and the averaged fingerprint was used as the characteristic fingerprint of the reference point. The comparison results under different noise standard deviations are shown in Fig. 10. 


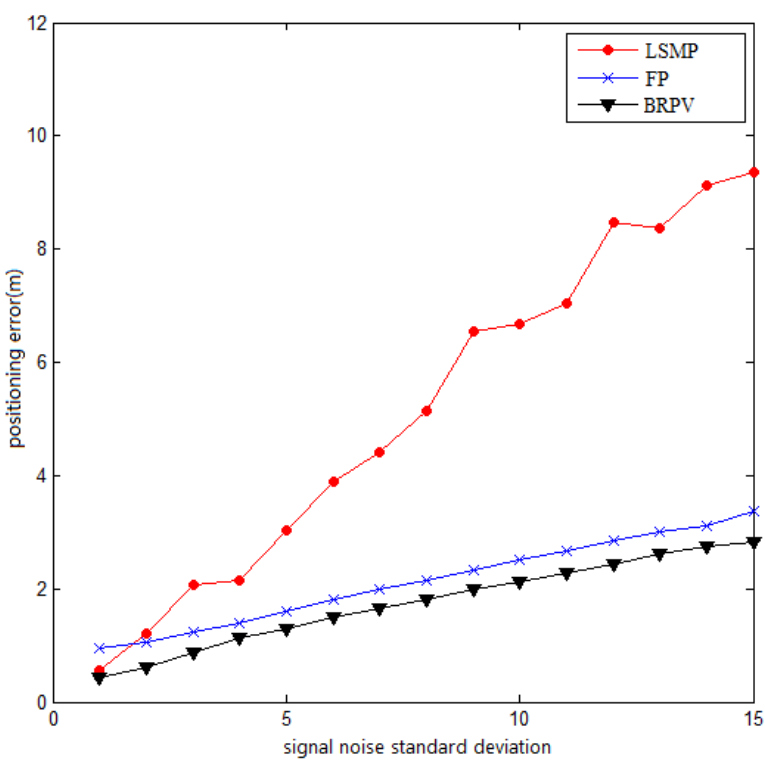

Fig. 10. Comparison of BRPV to LSMP and FP

From Fig. 10 it can be determined that 1) BRPV and FP have similar tolerance to noise, which is superior to the LSMP and 2) the localization accuracy of BRPV is slightly better than that of the FP and much better than that of the LSMP.

\subsection{The performance analysis of the BtPDR}

In this section, the theoretical simulation and test experiments of BtPDR in the real scene are described. The comparison algorithms include the basic PDR in [14], the proposed BRPV, the LSMP in [6], and the Kalman filter-based fingerprint positioning (KFFP) algorithm in [12].

\subsubsection{Theoretical simulation}

The theoretical simulation parameters in this paper are as follows: 1) four Bluetooth beacons in a localization scene were deployed and their coordinates were $(6,6),(0,12),(6$, $18)$ and $(0,24)$; 2$)$ the actual walking route was a $4.8 \mathrm{~m} \times 22.2 \mathrm{~m}$ rectangle and 3 ) considering that the number of received RSS samples in each real step was very low, in the simulation, each step only received one RSS sample. Moreover, the walking model of PDR is:

$$
\begin{aligned}
& L=L_{0}+A+X_{\sigma 1} \\
& \theta=\theta_{0}+B+X_{\sigma 2}
\end{aligned}
$$

Where $L_{0}$ and $\theta_{0}$ is the real length and heading of each step and $A$ and $B$ are constants used to simulate the fixed error caused by sensor accuracy limits, which were set to $A=0.1 \mathrm{~m}, B=3^{\circ}$ in simulation. $X_{\sigma 1}$ and $X_{\sigma 2}$ are zero mean Gaussian noises; $\sigma 1$ is the standard deviation of the step length, which was set to $\sigma 1=0.1$ and $\sigma 2$ is the standard deviation of heading, which was 
set to $\sigma 2=3.87$.

Since the simulation scene only had four Bluetooth beacons, according to Fig. 4 we can see that the range of votes in each region should be [4, 24]. Therefore, to ensure that the fusion results of BRPV and PDR are reliable, $\beta=18$ was set in the simulation. The fusion condition is that the number of votes of BRPV is larger than 18; otherwise, the localization results of BRPV are discarded and the localization results of PDR used as the final localization results.

Under the conditions of the theoretical experiment, the comparison results of different localization algorithms with different RSS noise errors are shown in Table 2 and Table 3.

Table 2. Comparison of algorithm performance $\left(\sigma^{2}=20\right)$

\begin{tabular}{|c|c|c|c|c|c|c|}
\hline Algorithm & $\begin{array}{c}\text { positioning } \\
\text { error/m }\end{array}$ & $\begin{array}{c}\mathbf{9 0 \%} \\
\text { positioning } \\
\text { error/m }\end{array}$ & $\begin{array}{c}\text { accuracy } \\
\text { within } \mathbf{~} \mathbf{m} \\
\mathbf{/ \%}\end{array}$ & $\begin{array}{c}\text { accuracy } \\
\text { within } \mathbf{2 m} \\
\mathbf{/ \%}\end{array}$ & $\begin{array}{c}\text { accuracy } \\
\text { within } \mathbf{3 m} \\
/ \mathbf{\%}\end{array}$ & $\begin{array}{c}\text { accuracy } \\
\text { within } \mathbf{5 m} \\
\mathbf{/ \%}\end{array}$ \\
\hline PDR & 3.17 & 4.79 & 21 & 38 & 56 & 94 \\
\hline BRPV & 2.06 & 4.97 & 26 & 68 & 79 & 90 \\
\hline LSMP & 4.96 & 9.76 & 16 & 32 & 40 & 61 \\
\hline KFFP & 1.87 & 3.78 & 34 & 62 & 73 & 95 \\
\hline BtPDR & 1.13 & 2.62 & 50 & 85 & 97 & 100 \\
\hline
\end{tabular}

Table 3. Comparison of algorithm performance $\left(\sigma^{2}=50\right)$

\begin{tabular}{|c|c|c|c|c|c|c|}
\hline Algorithm & $\begin{array}{c}\text { positioning } \\
\text { error/m }\end{array}$ & $\begin{array}{c}\mathbf{9 0 \%} \\
\text { positioning } \\
\text { error/m }\end{array}$ & $\begin{array}{c}\text { accuracy } \\
\text { within } \mathbf{~} \mathbf{m} \\
\mathbf{/ \%}\end{array}$ & $\begin{array}{c}\text { accuracy } \\
\text { within } \mathbf{2 m} \\
\mathbf{/ \%}\end{array}$ & $\begin{array}{c}\text { accuracy } \\
\text { within 3m } \\
\text { /\% }\end{array}$ & $\begin{array}{c}\text { accuracy } \\
\text { within } \mathbf{5 m} \\
\mathbf{/ \%}\end{array}$ \\
\hline PDR & 3.28 & 5.29 & 18 & 33 & 51 & 84 \\
\hline BRPV & 3.21 & 6.81 & 16 & 36 & 58 & 76 \\
\hline LSMP & 8.56 & 15.65 & 8 & 16 & 26 & 54 \\
\hline KFFP & 2.55 & 5.65 & 22 & 40 & 61 & 84 \\
\hline BtPDR & 1.66 & 2.72 & 35 & 66 & 97 & 100 \\
\hline
\end{tabular}

Table 2 and Table 3 show that BtPDR's positioning accuracy is the best because it has the smallest positioning error in these algorithms. Compared with PDR, BRPV, LSMP and KFFP, BtPDR has improved positioning accuracy by $64.3 \%, 45.1 \%, 77.2 \%$ and $39.6 \%$, respectively, in the case of a noise variance of 20 , and has improved positioning accuracy by $49.4 \%$, $48.3 \%, 80.6 \%$ and $34.9 \%$, respectively, in the case of a noise variance of 50 . With the increased noise variance of Bluetooth RSS, 1) the positioning accuracy of BRPV, LSMP and KFFP clearly became worse because they are related to Bluetooth RSS; 2) the positioning accuracy of PDR became small because PDR is unrelated to Bluetooth RSS and 3) the positioning error of BtPDR became large due to the increase of the positioning error of the BPRV. Note that the reason for the positioning error of BRPV and LSMP in Table 2 and Table 3 being significantly larger than the results in Table $\mathbf{1}$ is that each set step only 
received one RSS sample in the simulation, causing the RSS to have stronger fluctuation, thus increasing the positioning error. By contrast, each step in Table 1 received ten RSS samples and an average of RSS samples could be calculated to resist the RSS fluctuation.

\subsubsection{Actual location experiments}

Experiments were conducted in three actual scenarios including a separate indoor corridor, a separate office and a indoor corridor + office. A separate indoor corridor was used to test the performance of the localization algorithm in an ideal environment with few obstacles. A separate office was used to test the performance of the localization algorithm in a complex environment with lots of obstacles. A corridor + office environment was used to test the performance of the localization algorithm in a general environment.

The experimental field was a $42.2 \mathrm{~m} \times 21 \mathrm{~m}$ indoor environment as shown in Fig. 11. There were 12 Bluetooth beacons in the experiment field. The red stars in Fig. 11 represent the location of the Bluetooth beacons. The BRPV only used the 4 largest Bluetooth beacon RSS to locate the smartphone, hence, the range of the number of votes for each voting area in BRPV was [4, 24]. To enhance the reliability of BtPDR, $\beta=20$ was set in the actual scene. The Bluetooth beacons used a CC2541 Bluetooth 4.0 module, produced by Texas Instruments, with a radio transmission power of $0 \mathrm{dBm}$ and a launch rate of five times per second. The smartphone was a MX4 MeiZu, which has a built-in accelerometer, direction sensor, Bluetooth 4.0 chip and Android 4.4 OS.



Fig. 11. Experimental field map

To verify the robustness of BtPDR, three experimenters were invited to carry out the experiment. Each experimenter carried out four experiments for each experiment environment. Therefore, 36 experiments in total were carried out for three experimental environments. Since the sex, height, frequency and handheld mobile phone habits of each experimenter was different, the results can be used to test the robustness of BtPDR for different experimenters. 
a) indoor corridor + office

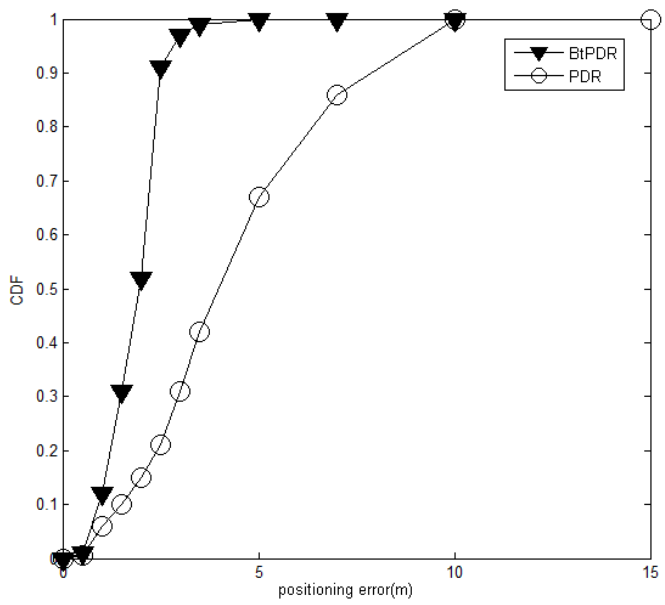

Fig. 12. The CDF of the positioning error

Experimental environment I was indoor corridor + office. The actual walking trajectory is represented by route 1 in Fig. 11. Route 1 had a distance of $82.7 \mathrm{~m}$ and passed indoor corridors and offices. The average absolute positioning errors of PDR and BtPDR were $4.23 \mathrm{~m}$ and $1.86 \mathrm{~m}$, respectively, and the cumulative distribution function (CDF) of the positioning error is shown in Fig. 12. Therefore, in the indoor corridor + office environment, BtPDR improved the positioning accuracy by up to $56 \%$, compared to PDR.

Fig. 13 compares location route results. As shown in Fig. 13(a), since the traditional PDR cannot accurately estimate or adaptively adjust the step size, the difference between positioning trajectory and actual trajectory is large. However, BtPDR can adaptively fuse the localization results of PDR and BRPV, adjusting the parameters of PDR when the localization results of BRPV are credible. Therefore, the positioning trajectory of BtPDR in Fig. 13(b) is clearly closer to the actual trajectory than that of PDR.

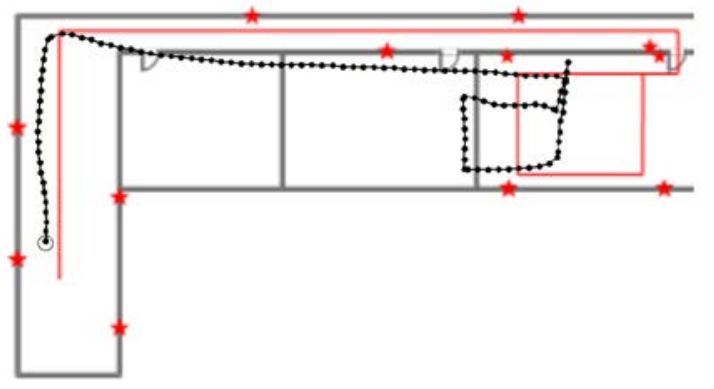

(a) The positioning trajectory of PDR

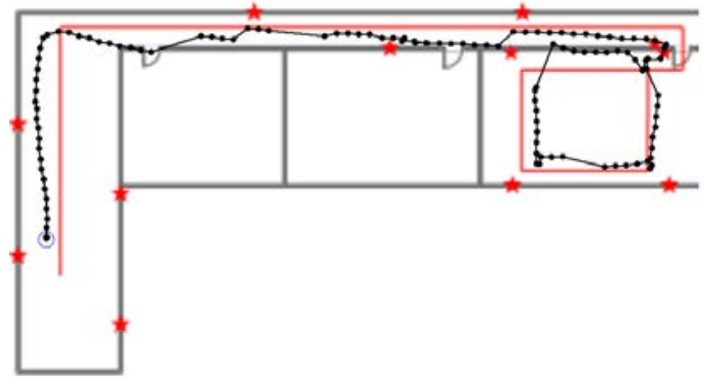

(b) The positioning trajectory of BtPDR

Fig. 13. Comparison of location route 
Fig. 14 compares cumulative error results. It can be seen that the cumulative error of PDR gradually increases with an increased number of steps and the error cannot be self-corrected. But BtPDR can correct the length of a forward step and the cumulative error will not increase with the increase of the number of steps.

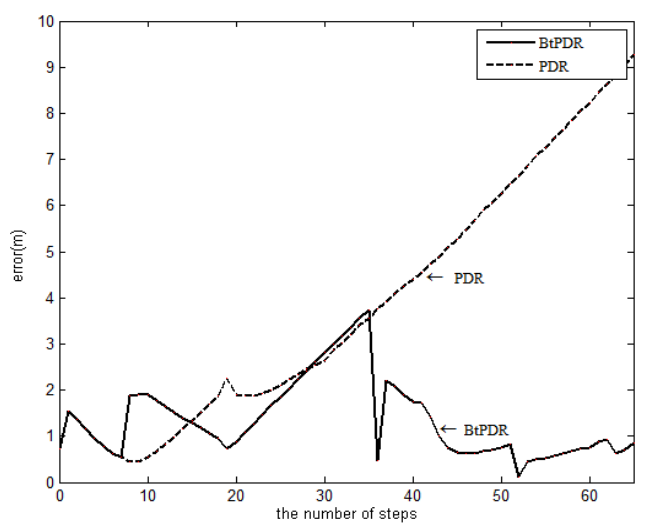

Fig. 14. Cumulative error distribution

b) Separate office environment

Experimental environment II consisted of only an office environment. The walking route is represented by route 2 in Fig. 11. Each experimenter walked along the green path two times for a total of $53.6 \mathrm{~m}$. The average absolute positioning errors of PDR and BtPDR were $2.59 \mathrm{~m}$ and $1.94 \mathrm{~m}$, respectively, and the positioning error CDF is shown in Fig. 15. Location route comparison results are shown in Fig. 16. It can be seen that, in the office environment, BtPDR improved the positioning accuracy by up to $25.1 \%$ compared to PDR. The positioning error of PDR in experiment environment II is clearly smaller than that of PDR in experiment environment I. The reasons for this are: 1) the walking route of experiment II was shorter than that of experiment I and PDR error increases as the walking path increases and 2) the walking route of experiment II was a complete circular route and the positioning error of PDR was offset due to the circular shape of route.

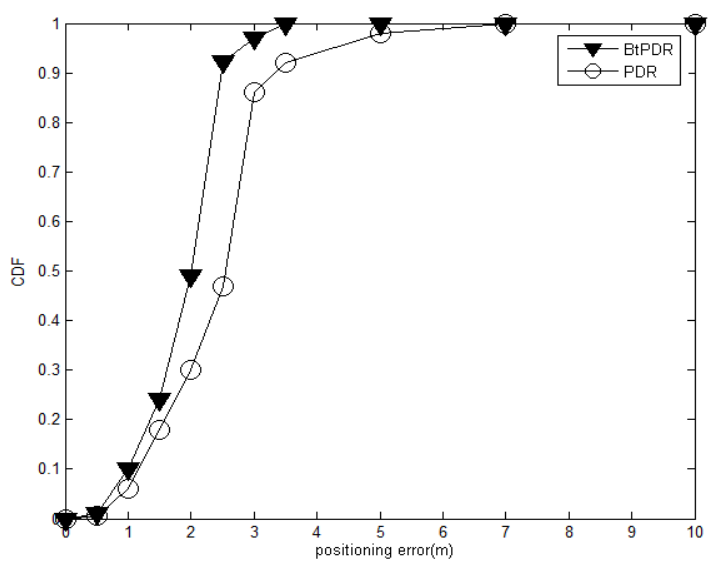

Fig. 15. The cumulative distribution function of the positioning error 


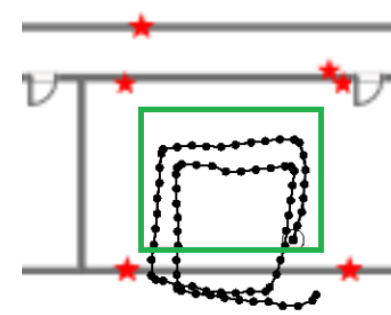

(a) positioning trajectory of PDR

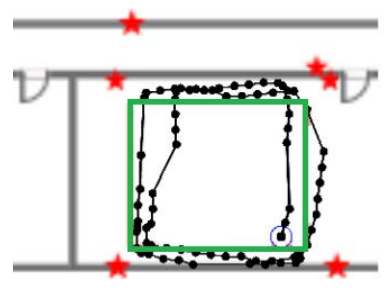

(b) positioning trajectory of BtPDR

Fig. 16. Comparison of location routes

c) Separate corridor environment

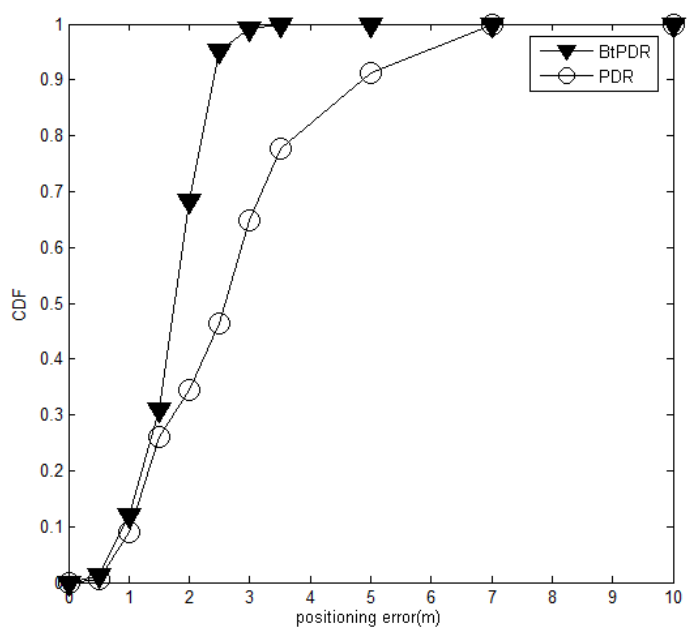

Fig. 17. The CDF of the positioning error in the corridor environment

Experimental environment III consisted of only a corridor environment. The walking route is represented by route 3 in Fig. 11. Each experimenter walked along the blue path two times for a total of $69.6 \mathrm{~m}$. The average absolute positioning errors of PDR and BtPDR were $2.97 \mathrm{~m}$ and 1.58m, respectively, and the positioning error CDF is shown in Fig. 17. Location route comparison results are shown in Fig. 18. It can be seen that, in the corridor environment, BtPDR improved the positioning accuracy by up to $46.8 \%$ compared to PDR. In addition, because the corridor environment is an ideal indoor environment with few obstacles, BRPV had more accurate localization results causing localization performance improvement of BtPDR. Therefore, BtPDR in the corridor environment has the best positioning accuracy. 


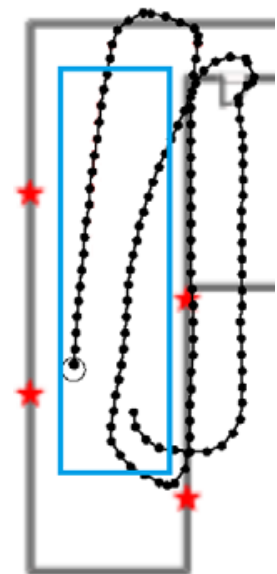

(a) positioning trajectory of PDR

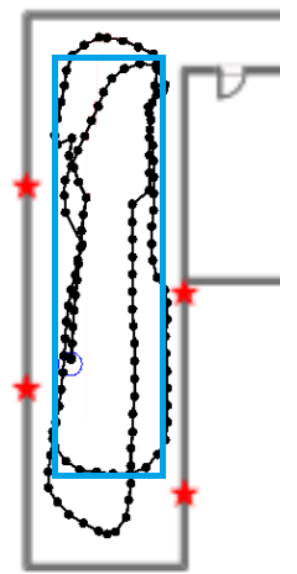

(b) positioning trajectory of BtPDR

Fig. 18. Comparison of location routes in the corridor environment

\subsubsection{The Latency of BtPDR}

Latency is another key indicator of the positioning system in practice. An average person walks about 95 125 steps per minute, meaning their speed is about 480 630ms per step. Through the experiment, we found that BtPDR requires about 30ms of processing time for each step. Therefore, this study argues that, in practical application, the latency of BtPDR meets real-time positioning.

\subsection{Compared with other indoor fusion localization algorithms}

Because Bluetooth and PDR-based fusion localization methods are seldom seen in the literature and most fusion localization methods are based on WLAN and PDR, the indoor localization algorithms in [16-18] were chosen for comparison. These algorithms were chosen because their experimental environments are like this study's experimental environment and they are relatively new fusion algorithms. The work in [16] and [17] are fusion localization algorithms based on PDR and WLAN fingerprints. The experiment settings in [16] included a HTC Hero with Android 1.5 OS mobile terminal; 24 WLAN beacons in the positioning region; a fingerprint reference point deployed every three meters creating 183 reference points; and for each reference point, 100 fingerprint acquisitions were carried out. In [16], the average positioning errors of three test paths were $2.76 \mathrm{~m}, 2.57 \mathrm{~m}$ and $2.81 \mathrm{~m}$, which is worse than the results of BtPDR. The experiment settings in [17] included a Samsung Galaxy S2 with Android 2.2 OS mobile terminal; 10 WLAN beacons in the positioning region; a fingerprint reference point deployed every two meters creating 72 reference points; and for each reference point, 500 fingerprint acquisitions were carried out. 
In [17], the experimental results showed that $90 \%$ of the positioning error was less than $4 \mathrm{~m}$ and the average positioning error was $1.9 \mathrm{~m}$. The average positioning error in [17] is like that of BtPDR, but a 90\% node localization error is less than the results of BtPDR. The work in [18] used PDR and map information to realize fusion localization. The experiment settings in [18] included a sensor worn on the waist and map information needed to detect the correctness of trajectory. The average positioning error of the two experiments was $1.4 \mathrm{~m}$ and $1.7 \mathrm{~m}$, respectively. The reasons that the experimental results in [18] are superior to BtPDR are: compared to a sensor held in the hand, the sensor worn on the waist can reduce the volatility of the collected data and the experiment used extra map information and a complex particle filter to refine PDR results.

In summary, compared to previous literature, the proposed BtPDR not only provides accurate indoor localization but also eliminates the work for acquisition of offline fingerprint or map information. Moreover, to validate the robustness of BtPDR, three testers were chosen to carry out the experiment in three different experimental scenarios. The work in [16-18] did not mention conducting a robustness experiment and the indoor environment was an ideal corridor environment. Therefore, this study suggests that the robustness of BtPDR is superior to that of the algorithms in [16-18].

\section{Conclusion}

This study presented an easy to implement approach, BtPDR, for indoor localization and trajectory tracking that combines Bluetooth RSS with PDR. First, the initial position of a smartphone was localized with BRPV. Second, while the smartphone was moving, it was tracked with PDR and BRPV at the same time. Last, localization results of PDR and BRPV were fused and parameters of PDR were adaptively modified. Experiment results show that the positioning error of BtPDR is less than $2 \mathrm{~m}$, which is significantly better than that of PDR and BRPV. Moreover, experiment results also show that, compared to the WLAN fingerprint and PDR-based fusion indoor localization approaches, BtPDR has better positioning accuracy and does not need the offline workload of a fingerprint algorithm. Therefore, the proposed BtPDR is accurate, stable and practical. This study is not without limitations. Due to the limitation of experimental conditions, localization experiments were not carried out in a wide range of indoor areas; the RSS error due to the shadow effect of body occlusion was not considered and considering the recent data precision enhancement techniques of IMU sensors to further improve the localization accuracy of PDR would be beneficial. These shortcomings will be addressed in future work. 


\section{REFERENCES}

[1] Liu Y, Yang Z, Wang X, et al. "Location, localization, and localizability[J]," Journal of Computer Science and Technology, 25(2), 274-297, 2010. Article (CrossRef Link)

[2] Harle R. "A survey of indoor inertial positioning systems for pedestrians[J]," IEEE Communications Surveys \& Tutorials, 15(3), 1281-1293, 2013. Article (CrossRef Link)

[3] Yohan Chon, Hojung Cha, "LifeMap: A Smartphone-Based Context Provider for Location-Based Services[J],” IEEE Pervasive Computing, 10(2), 58-67, 2011.

Article (CrossRef Link)

[4] Z. Hui-Qing, S. Xiao-Wei, C. Lu-Guang and D. Gui-Hua. "A new indoor location technology using back propagation neural network and improved centroid algorithm," in Proc. of 31st Chinese Control Conference (CCC), pp. 5460-5463, Hefei, China, 2012.

[5] A. Fink, H. Beikirch. "Radio-based human tracking for large indoor environments using distributed centroid location estimation[C]," in Proc. of IEEE 7th International Conference on Intelligent Data Acquisition and Advanced Computing Systems (IDAACS), pp. 442-449, Berlin, Germany, 2013.

[6] Waadt A E, Kocks C, Wang S, et al. "Maximum likelihood localization estimation based on received signal strength[C]," in Proc. of 3rd International Symposium on Applied Sciences in Biomedical and Communication Technologies (ISABEL), IEEE, pp. 1-5, Roma, Italy, 2010. Article (CrossRef Link)

[7] Sichun Wang, Jackson B.R., Rajan S, Patenaude, F. "Received Signal Strength-Based Emitter Geolocation Using an Iterative Maximum Likelihood Approach[C]," in Proc. of Military Communications Conference(MILCOM '13), pp:68-72, San Diego, USA, 2013. Article (CrossRef Link)

[8] Wuk Kim, Jang Gyu Lee, Gyu-In Jee. "The interior-point method for an optimal treatment of bias in trilateration location[J],” IEEE Transactions on Vehicular Technology, 55(4): 1291-1301, 2006. Article (CrossRef Link)

[9] Gentile, C. "Distributed sensor location through linear programming with triangle inequality constraints[C]," in Proc. of IEEE International Conference on Communications(ICC '06), pp. 4020-4027, Istanbul , Turkey, 2006. Article (CrossRef Link)

[10] Au W S A, Feng C, Valaee S, et.al. "Indoor tracking and navigation using received signal strength and compressive sensing on a mobile device[J]," IEEE Transactions on Mobile Computing, 12(10), 2050-2062, 2013. Article (CrossRef Link)

[11] Fang S H, Lin T N. "Principal component localization in indoor WLAN environments[J]," IEEE Transactions on Mobile Computing, 11(1), 100-110, 2012. Article (CrossRef Link)

[12] ZHAO Yongxiang, ZHOU Huaibei, CHEN Miao, WEN Bin. "Application of Kalman Filter in Indoor Positioning System for Real -Time Tracking [J],” Journal of Wuhan University, 25(6), 696-700, 2009. 
[13] L. Zhang, X. Liu, J. Song, C. Gurrin and Z. Zhu. "A Comprehensive Study of Bluetooth Fingerprinting-Based Algorithms for Localization[C],” in Proc. of 27th International Conference on Advanced Information Networking and Applications Workshops (WAINA), pp. 300-305, Barcelona, Spain, 2013. Article (CrossRef Link)

[14] Jimenez A.R, Seco F, Prieto C, Guevara J. “A comparison of Pedestrian Dead-Reckoning algorithms using a low-cost MEMS IMU [C]," in Proc. of IEEE International Symposium on Intelligent Signal Processing(WISP ’09), pp:37-42, Budapest, Hungary, 2009.

Article (CrossRef Link)

[15] Radu V, Marina M K. "HiMLoc: Indoor smartphone localization via activity aware Pedestrian Dead Reckoning with selective crowdsourced WiFi fingerprinting[C],” in Proc. of International Conference on Indoor Positioning and Indoor Navigation (IPIN '13), pp:1-10, Montbeliard-Belfor, French, 2013. Article (CrossRef Link)

[16] Lyu-Han Chen, Wu E.H.-K, Ming-Hui Jin, Gen-Huey Chen. "Intelligent Fusion of Wi-Fi and Inertial Sensor-Based Positioning Systems for Indoor Pedestrian Navigation [J],” IEEE Sensors Journal, 14(11): 4034-4042, 2014. Article (CrossRef Link)

[17] Bonhyun Koo, Sangwoo Lee, Sunwoo Kim, Cheonsig Sin, "Integrated PDR/fingerprinting indoor location tracking with outdated radio map[C]," in Proc. of 2014 IEEE Region 10 Conference, pp. 1-5, Bangkok, Thailand, 2014.

[18] Perttula A, Leppakoski H, Kirkko-Jaakkola M, Davidson P, Collin J, Takala J. “Distributed Indoor Positioning System With Inertial Measurements and Map Matching[J],” IEEE Transactions on Instrumentation and Measurement, 63(11), 2682-2695. 2014.

Article (CrossRef Link)

[19] Wonho Kang, Youngnam Han. "SmartPDR: Smartphone-Based Pedestrian Dead Reckoning for Indoor Localization[J],” IEEE Sensors Journal, 15(5): 2906-2917, 2015. Article (CrossRef Link)

[20] Zuwei Yin, Chenshu Wu, Zheng Yang, Yunhao Liu. "Peer-to-Peer Indoor Navigation using Smartphones[J],” IEEE Journal on Selected Areas in Communications, 35(5): 1141-1153, 2017. Article (CrossRef Link)

[21] Rai A, Chintalapudi KK, Padmanabhan VN, Sen R. "Zee: zero-effort crowdsourcing for indoor localization[C],” in Proc. of ACM MobiCom’12, pp. 293-304, Istanbul, Turkey, 2012.

[22] Chenshu Wu, Zheng Yang, Yunhao Liu. "Smartphones Based Crowdsourcing for Indoor Localization[J],” IEEE Transactions on Mobile Computing, 14(2): 444-457, 2015.

Article (CrossRef Link)

[23] Kaishun Wu, Jiang Xiao, Youwen Yi, et al. “CSI-Based Indoor Localization[J],” IEEE Transactions on Parallel \& Distributed Systems, 24(7), 1300-1309, 2013.

Article (CrossRef Link)

[24] A. Ryu, M. Nelson, A. Mehtala, T. Nyberg and G. Xiong. "Non-GPS positioning sensor network in social manufacturing[C]," in Proc. of 2016 IEEE International Conference on Service Operations and Logistics, and Informatics (SOLI), pp. 47-52. Beijing, China, 2016.

Article (CrossRef Link) 
[25] Fard H K, Yuanzhu Chen, Kyung Kook Son. "Indoor positioning of mobile devices with agile iBeacon deployment[C]," in Proc. of IEEE 28th Canadian Conference on Electrical and Computer Engineering (CCECE '15), pp. 275-279, Halifax, Canada, 2015.

[26] C C Pu, W Y Chung. "Mitigation of multipath fading effcts to improve indoor RSSI performance[J],” IEEE Sensors Journal, 8(11), 1884-1886, 2008. Article (CrossRef Link)

[27] Z Fang, Z Zhao, D Geng, Y Xuan, L Du, X Cui. "RSSI variability characterization and calibration method in wireless sensor network[C]," in Proc. of IEEE International Conference on Information and Automation (ICIA '10), pp. 1532-1537, Harbin, China, 2010.

Article (CrossRef Link)

[28] V Daiya, J Ebenezer, S A V S Murty, B Raj. "Experimental analysis of RSSI for distance and position estimation[C]," in Proc. of IEEE International Conference on Recent Trends in Information Technology (ICRTIT '11), pp.1093-1098, Tamil Nadu, India, 2011. Article (CrossRef Link)

[29] F. Yin, Ang Li, A. M. Zoubir, C. Fritsche and F. Gustafsson. "RSS-based sensor network localization in contaminated Gaussian measurement noise[C]," in Proc. of 5th International Workshop on Computational Advances in Multi-Sensor Adaptive Processing (CAMSAP), pp. 121-124. St. Martin, French, 2013. Article (CrossRef Link)

[30] Luan V N and Hung M L. "A Human Foot Motion Localization Algorithm Using IMU[C]," in Proc. of 2016 American Control Conference (ACC), pp. 4379-4384, Boston, USA, 2016. 


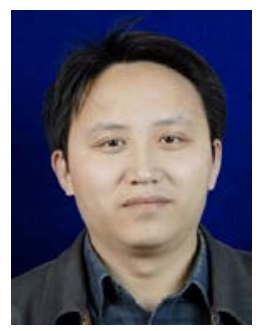

Yingbiao Yao is with the College of communication Engineering, Hangzhou Dianzi University (HDU), Hangzhou, China, as a professor. He received the M. Eng. degree in Communication and Information System from Xi'an Shiyou University, Xi'an, China, in 2003, He received Ph.D. degree major in Communication and Information System from Zhejiang University (ZJU), Hangzhou, China, in 2006. Since 2006, he has been with the College of communication Engineering, HDU. During 2011-2012, he served as a research scholar with the Electrical and Computer Engineering Department, Rensselaer Polytechnic Institute, Albany, NY, USA. Currently, he is working as a professor in HDU. Dr. Yao's research interests emphasize on computer architecture, media signal processing, wireless localization, etc.

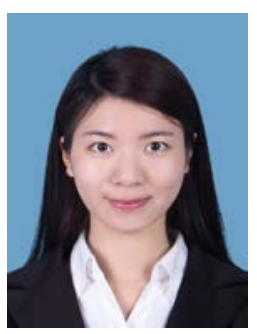

Qiaojing Bao was born in 1992. She received her B.S.degree in Electronic and Information Engineering from Zhejiang University of Science and Technology, Hangzhou, China, 2015. She is with the College of Telecommunication Engineering, Hangzhou Dianzi University (HDU), Hangzhou, China, as postgraduate student. Currently, she is working toward her M. Eng. degree in Electronics and Communications Engineering in HDU. Her research interests include indoor positioning and edge caching .

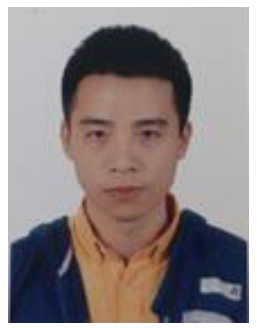

Han Qi is with the College of communication Engineering, Hangzhou Dianzi University (HDU), Hangzhou, China, as postgraduate student. He received the B. Eng. degree in Communication Engineering from HDU, Hangzhou, China, in 2014. Currently, he is working toward his M. Eng. degree in Electronics and Communications Engineering in HDU. His research interest is indoor positioning.

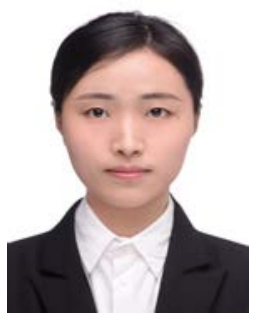

Ruili Yao is with the College of communication Engineering, Hangzhou Dianzi University (HDU), Hangzhou, China, as a postgraduate student. She received the B. Eng. degree in Communication Engineering from Shandong University of Technology, Shandong, China, in 2015. Currently, she is working toward her M. Eng. degree in Electronics and Communications Engineering in HDU. Her research interest is indoor positioning. 


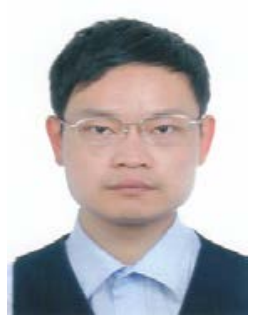

Xiaorong $\mathbf{X u}$ is with the College of communication Engineering, Hangzhou Dianzi University (HDU), Hangzhou, China, as associate professor. He received the B. Eng. degree in Communication Engineering and M. Eng. degree in Communication and Information System from HDU, Hangzhou, China, in 2004 and 2007, respectively. He received Ph.D. degree major in Signal and Information Processing from Nanjing University of Posts and Telecommunications (NJUPT), Nanjing, China, in 2010. Previously, from 2011 to 2013, he was working as a postdoctoral researcher in the Institute of Information and Communication Engineering, Zhejiang University (ZJU), Hangzhou, China. During 2013-2014, he served as a research scholar with the Electrical and Computer Engineering Department, Stevens Institute of Technology (SIT), Hoboken, NJ, USA. Currently, he is working as a associate professor in HDU. Dr. Xu's research interests emphasize on energy efficiency in cooperative communications, energy efficiency and PHY security in Cognitive Radios (CR), as well as Compressive Sensing (CS) theory, etc.

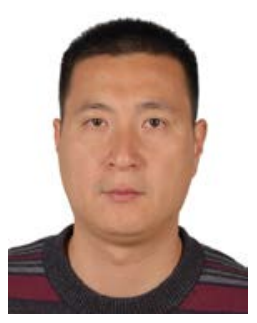

Junrong Yan received M. Eng. degree in Communication and Information System from Kunming university of science and technology (KMUST), Kunming, China, in 2006. He received Ph.D. degree major in Information networks from Nanjing University of Posts and Telecommunications (NJUPT), Nanjing, China, in 2010. Since 2010, he has been with the College of communication Engineering, Hangzhou Dianzi University (HDU), Hangzhou, China, as a lecturer. During 2011-2013, he served as a postdoctoral research fellow with the Institute of Information and Communication Engineering, Zhejiang University (ZJU), Hangzhou, China. During 2017, he served as a research scholar with the School of Electrical and Computer Engineering, Oregon State University, Corvallis, OR, USA. Currently, he is still working in HDU. Currently, Dr. Yan's research interests emphasize on wireless communication, Software definition network, etc. 